\title{
FATORES DE RISCO PREDOMINANTES NA POPULAÇÃO COM SÍNDROME CORONARIANA AGUDA
}

\section{PREVAILING RISK FACTORS IN THE POPULATION WITH ACUTE CORONARY SYNDROME}

\author{
Amanda Francielle Santos, Rafaela Ribeiro Machado, Míriam Geisa V. Menezes
}

Faculdade Estácio de Sergipe

\begin{abstract}
The acute coronary syndrome has been responsible for a great number of deaths around the world, and in Brazil it presents a high prevalence, resulting in a necessary evaluation of its risk factors in the population. This research aims to describe the most prevailing risk factors in individuals with acute coronary syndrome through the literature. It is an integrative bibliographic and descriptive research with a qualitative approach, carried out from national articles published from 2013 to 2017, deriving from the electronic data basis BIREME, LILACS and SCIELO, which deal with the present subject. The analysis of the studies shows that the most prevailing non-modifiable risk factors were age, male gender and family history. The modifiable risk factors were high blood pressure, diabetes, dyslipidemia, sedentariness, obesity and smoking. This study concludes that most part of the studied population with acute coronary syndrome suffers with a greater influence of modifiable risk factors. The most frequent ones were high blood pressure, followed by smoking. It is necessary that the healthcare professionals evaluate the most prevailing risk factors in the as- sisted population, in order to make an addressed and effective assistance.
\end{abstract}

Resumo

A Síndrome Coronária Aguda tem sido responsável por um grande número de morte no mundo e no Brasil ela tem uma alta prevalência, sendo necessário que seus fatores de risco na população sejam avaliados. Objetiva-se com essa pesquisa descrever através da literatura os fatores de risco mais prevalentes em indivíduos com síndrome coronariana aguda. Trata-se de uma pesquisa descritiva bibliográfica integrativa de abordagem qualitativa, realizada a partir de artigos nacionais publicados entre 2013 a 2017, oriundos das bases de dados eletrônicas BIREME, LILACS e SCIELO que abordassem o tema pesquisado. A análise dos estudos mostra que os fatores de risco não modificáveis mais prevalentes foram idade, sexo masculino $e$ história familiar. Já os fatores de risco modificáveis foram hipertensão arterial, diabetes, dislipidemia, sedentarismo, obesidade e tabagismo. Conclui-se com esse estudo que a maior parte da população estudada com síndrome coronariana aguda sofre com maior influência de fatores de risco modificáveis. Os fatores de risco modificáveis mais freqüentes nos estudos foram a hipertensão arterial, seguida de tabagismo. É necessário que os profissionais da saúde avaliem os fatores de risco mais prevalentes na população a ser assistida, visando realizar uma assistência direcionada e consequentemente mais eficaz.

Palavras chave: fatores de risco; síndrome coronariana aguda; prevalência.
Key words: fatores de risco; síndrome coronariana aguda; prevalência. 
Introdução

A síndrome coronariana aguda é o principal motivo de morte entre a população no Brasil e no mundo $^{1}$. Em 2013, 29,9\% dos óbitos foram por doenças do aparelho circulatório, sendo $47,3 \%$ doenças isquêmicas do coração ${ }^{2}$.

A síndrome coronariana aguda (SCA) é um termo amplo, utilizado para descrever um grupo de patologias causadas pela obstrução das artérias coronárias e abrange diversos grupos de sintomas compatíveis com isquemia miocárdica aguda, causada pelo desequilíbrio entre o suprimento e a demanda de oxigênio no miocárdio. A SCA engloba o infarto agudo do miocárdio que pode ser com supra desnivelamento do segmento ST e sem supra desnivelamento do segmento ST, além da Angina Instável ${ }^{3,4}$

São considerados como fatores de risco para as doenças cardiovasculares: sexo, idade, raça, genética, fumo, dieta inadequada, inatividade física, ingestão de álcool, hipertensão arterial, obesidade, hiperlipidemia, diabetes mellitus e características socioeconômicas-culturais ${ }^{5}$.

Existem dois grupos de fatores de risco, os fatores não modificáveis quando os sujeitos não podem controlar ou alterar e desse modo não podem ser modificados pelas alterações do estilo de vida e os modificáveis que podem ser controlados ou prevenidos e devem ser pauta de educação em saúde dos profissionais de saúde. Os fatores de risco atuam em conjunto e quanto maior o acumulo delas no indivíduo maior é a possibilidade do desenvolvimento de doenças coronarianas $^{6}$.

Os indivíduos que apresentam a aglomeração de quatro ou mais fatores de risco para doenças cardiovasculares têm uma probabilidade maior de desenvolver doença isquêmica do coração quando comparados àqueles com nenhum ou um fator. É importante preservar a saúde e manter independência ao envelhecer. A idade avançada, por si só, aumenta os riscos de doenças crônicas, havendo destaque para as patologias cardiovasculares. Porém, o progresso dos danos a saúde acomete de forma diferente os indivíduos, há uma menor frequência e gravidade naqueles com trajeto e cotidiano de vida mais saudáveis ${ }^{7}$.

Estudar os fatores de risco relacionados a doença cardiovascular é uma etapa essencial para avaliar o impacto real destes na ocorrência de doenças em nossa população ${ }^{8}$. A pesquisa justifica-se pela necessidade de um estudo que reúna os dados presentes na literatura acerca de fatores de risco na tentativa de identificar os mais prevalentes, visando estimular os profissionais da saúde a adotarem uma atenção especial a esses.

Objetiva-se com essa pesquisa descrever através da literatura os fatores de risco mais prevalentes em indivíduos com síndrome coronariana aguda.

\section{Metodologia}

Estudo descritivo com abordagem qualiquantitativa. Foram seguidas as seguintes etapas: identificação da questão de pesquisa; definição dos critérios de inclusão e exclusão de estudos; categorização e avaliação dos estudos, extração e interpretação dos resultados e síntese do conhecimento. A pergunta que embasou o levantamento dos dados da pesquisa foi: Quais são os fatores de risco mais prevalentes em indivíduos com síndrome coronariana aguda?

A busca foi realizada nas bases de dados eletrônicas BIREME, LILACS, e SciELO durante os meses de junho a julho de 2017. Selecionaram-se para análise artigos com a publicação nos últimos cinco anos. A detecção ocorreu mediante a utilização dos seguintes descritores: Síndrome coronariana aguda. Perfil de saúde. Fatores de risco. Combinando-os com o operador boleano "AND". Critérios de inclusão do estudo: artigos apresentados com texto na íntegra; escritos em português; publicados entre 2013 a 2017, que abordassem os termos citados anteriormente. Foram excluídos do estudo: monografias; artigos de revisão; anais de eventos; dissertações; teses; livros; além de artigos fora do período estabelecido no estudo. Após a seleção foram analisados 08 artigos e posteriormente submetidos à produção de fichamentos dos conteúdos relevantes à temática.

A caracterização dos estudos e os fatores de risco relatados nos mesmos estão apresentados em tabela e os fatores que obtiveram uma porcentagem a partir de $30 \%$ nos estudos têm sua prevalência exposta em gráfico. 
Fluxograma 1- Fluxo da informação com as diferentes fases de busca de artigos para revisão integrativa: segue o modelo PRISMA adaptado

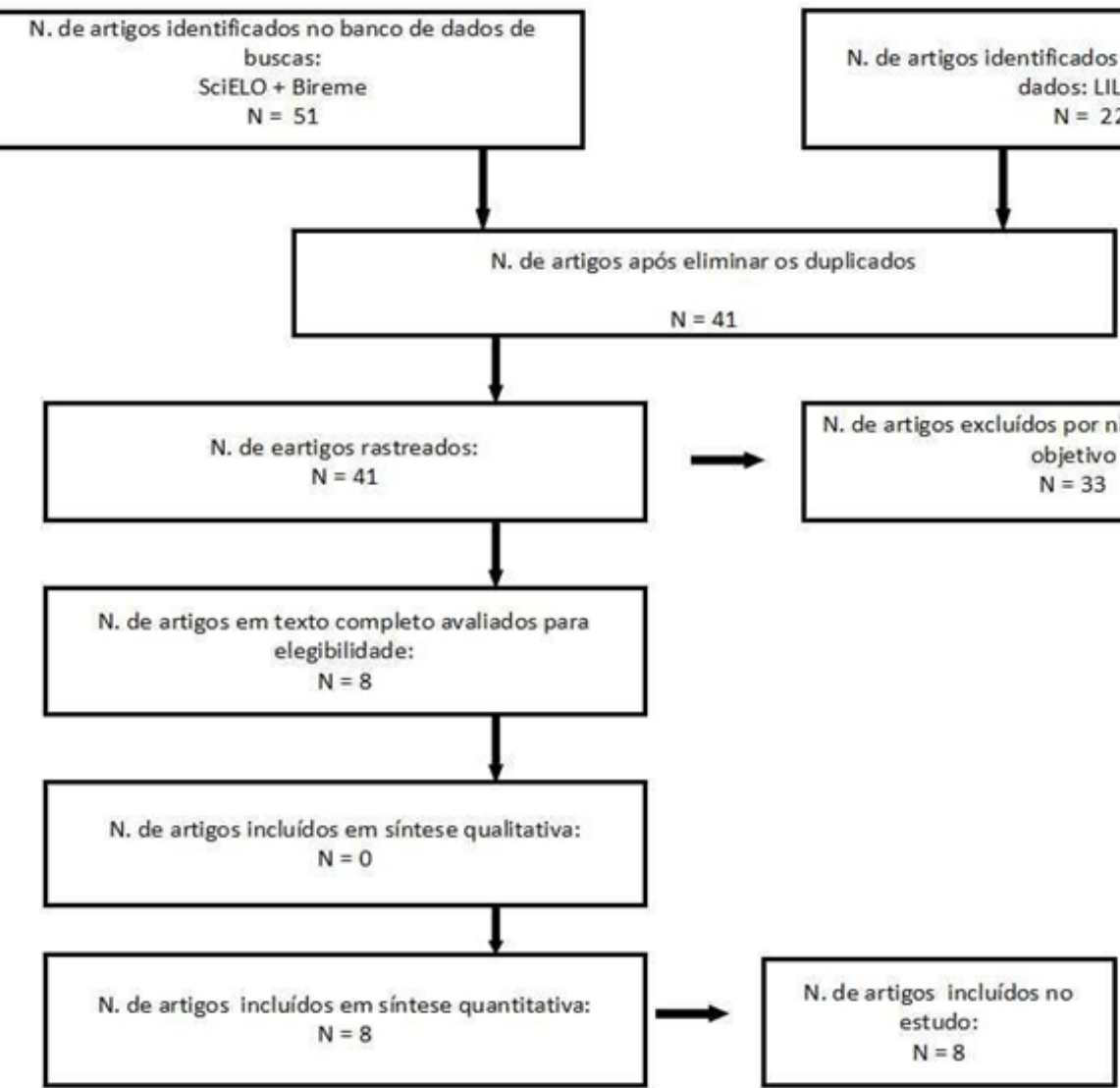

\section{Resultados e Discussões}

Dentre os fatores de risco não modificáveis, associados ao desenvolvimento de doenças cardiovasculares (DCV), podem ser citados idade acima de 55 anos, história familiar de DCV, sexo masculino e etnia para algumas afeç̧ões. Constitui-se como fatores de risco modificáveis a dislipidemia, tabagismo, hipertensão arterial sistêmica, inatividade física, obesidade, diabetes Mellitus, dietas não saudáveis e estresse psicossocial $^{9}$.

A análise dos estudos mostra que os fatores de risco não modificáveis mais prevalentes foram idade, sexo masculino e história familiar. Já os fatores de risco modificáveis foram hipertensão arterial, diabetes, dislipidemia, sedentarismo, obesidade e tabagismo (Quadros 1 a 2 e Figura 1).

O estudo mostra que a maior parte da população estudada com síndrome coronariana aguda sofre com maior influência de fatores de risco modicáveis. Evidenciando que é possível reduzir os índices através da estimulação à adoção de hábitos saudáveis a população.

Há a necessidade de implantação de políticas de saúde voltadas para a prevenção primária ou secundária com alvo, principalmente, em mudanças no estilo de vida, como a cessação do tabagismo, a prática regular de atividades físicas e a dieta equilibrada. Essas ações têm um baixo custo e um grande potencial para diminuição de novos casos de infarto ${ }^{10}$.

Os fatores de risco modificáveis mais frequentes nos estudos foram a hipertensão arterial, seguida do tabagismo. A Hipertensão Arterial de forma progressiva causa lesões nos vasos sanguíneos com consequentes modificações de órgãos alvos como coração ${ }^{11}$. A baixa renda, a dificuldade de acesso aos serviços de saúde e a maior prevalência dos fatores de risco, favorecem tanto o baixo controle, quanto a morte de hipertensos ${ }^{10}$.

Existem estudos que evidenciam o risco coronariano com a utilização do tabaco por aumentar a ação inflamatória pela elevação dos 
níveis plasmáticos de TNF $\alpha$ e PCR ${ }^{12}$. Os índices do tabaco como fator de risco estão reduzindo, em decorrência do declínio do seu consumo no Brasil $^{13}$. Em 2014, um decreto presidencial que proibiu o consumo de produtos fumígenos em locais coletivos fechados, impediu as propagandas de cigarro, e também aumentou as advertências nas embalagens e os impostos relacionados ao produto ${ }^{14}$ A avaliação da escolaridade e da situação socioeconômico não ocorreu em todos os estudos avaliados, mesmo estudos mostrando que são fatores relevantes. A renda e a escolaridade são relacionadas aos fatores de risco, pois influenciam no nível de autocuidado do indivíduo ${ }^{15}$. É fundamental que sejam investidos em estratégias sociais apropriadas de fácil entendimento pela população e de forma abrangentes para abrandar as diferenças socioeconômicas e dessa forma diminuir as enfermidades e recuperar a saúde dos mais vulneráveis ${ }^{16}$.

A maneira mais eficaz de diminuir o impacto das patologias cardiovasculares na comunidade é o desenvolvimento de ações para a prevenção e tratamento dos fatores de risco. O profissional da saúde deve conhecer os fatores de risco que desencadeiam a síndromes coronarianas agudas, e quais são as mais prevalentes dentro da sua área de atuação, a fim de atuar de forma mais eficaz no desenvolvi- mento de ações capazes de minimizar a morbidade e mortalidade por doença cardiovascular $^{17}$

Quadro 1 - Caracterização dos estudos sobre síndrome coronariana aguda e seus fatores de risco, Brasil, 2017.

\begin{tabular}{|c|c|c|c|c|}
\hline Código & Autor/ano & Local & Desenho & Amostra \\
\hline 01 & $\begin{array}{c}\text { JESUS; } \\
\text { CAMPELO; } \\
\text { SILVA, 2013. }\end{array}$ & $\begin{array}{l}\text { Piauí, } \\
\text { Brasil. }\end{array}$ & $\begin{array}{l}\text { Descritivo, analítico e } \\
\text { retrospective }\end{array}$ & $\begin{array}{l}240 \\
\text { Prontuários }\end{array}$ \\
\hline 02 & $\begin{array}{l}\text { ALMEIDA et } \\
\text { al. } 2014\end{array}$ & $\begin{array}{l}\text { Pernambuco, } \\
\text { Brasil. }\end{array}$ & Descritivo Transvesal & $\begin{array}{c}927 \\
\text { Individuos }\end{array}$ \\
\hline 03 & $\begin{array}{l}\text { BRUNORi et } \\
\text { al. } 2014\end{array}$ & $\begin{array}{l}\text { São Paulo, } \\
\text { Brasil. }\end{array}$ & Descritivo transversal & $\begin{array}{l}150 \\
\text { Individuos }\end{array}$ \\
\hline 04 & $\begin{array}{l}\text { ANDRADE at } \\
\text { al. } 2015\end{array}$ & Brasil. & $\begin{array}{l}\text { Descritivo, } \\
\text { retrospectivo. }\end{array}$ & $\begin{array}{c}489 \\
\text { Individuos }\end{array}$ \\
\hline 05 & $\begin{array}{c}\text { SILVA et al. } \\
2015\end{array}$ & Minas Gerais & $\begin{array}{c}\text { Observacional de } \\
\text { coorte }\end{array}$ & $\begin{array}{c}40 \\
\text { Indivíduos }\end{array}$ \\
\hline 06 & $\begin{array}{l}\text { SANTOS et } \\
\text { al. } 2015\end{array}$ & $\begin{array}{l}\text { São Paulo, } \\
\text { Brasil }\end{array}$ & Transversal e analítico. & $\begin{array}{c}84 \\
\text { Prontuários }\end{array}$ \\
\hline 07 & $\begin{array}{l}\text { ARAÚJO et } \\
\text { al.2016 }\end{array}$ & $\begin{array}{l}\text { Bahia, } \\
\text { Brasil. }\end{array}$ & $\begin{array}{l}\text { Descritivo, retrospectivo, } \\
\text { de análise documental }\end{array}$ & $\begin{array}{l}106 \\
\text { Prontuário }\end{array}$ \\
\hline 08 & $\begin{array}{l}\text { MERTINS et } \\
\text { al. } 2016\end{array}$ & $\begin{array}{l}\text { Rio Grande do } \\
\text { Sul, Brasil. }\end{array}$ & Descritivo transversal. & $\begin{array}{c}48 \\
\text { Individuos }\end{array}$ \\
\hline
\end{tabular}


Quadro 2 - Doenças e fatores de risco relatados nos estudos sobre síndrome coronariana aguda e seus fatores de risco, Brasil, 2017.

\begin{tabular}{|c|c|}
\hline Código & Fatores de risco \\
\hline 01 & $\begin{array}{l}\text { Sexo masculino (56,2\%); faixa etária média de } 61,92 \pm 10,6 \text { anos; ensi- } \\
\text { no fundamental incompleto }(34,5 \%) \text {; hipertensão arterial } 84,6 \% \text {; dislipi- } \\
\text { demias } 7,5 \% \text {; diabetes }(29,6 \%) \text {; tabagismo }(35,4 \%) \text {; etilismo }(6,7 \%) \text {. }\end{array}$ \\
\hline 02 & $\begin{array}{l}\text { Sexo masculino } 60,0 \% ; \quad 50 \% \text { de cor branca; etilismo em mulheres } \\
(21,2 \%) \text { e em homens }(24,8 \%) \text {; tabagismo em mulheres }(16,2 \%) \text { e em } \\
\text { homens( } 24,1 \%) \text {; sedentarismo em mulheres( } 86,3 \%) \text { e em homens } \\
(66,8 \%) \text {; HAS em mulheres }(74,1 \%) \text { e em homens }(72,7 \%) \text {; diabetes em } \\
\text { mulheres }(37,2 \%) \text { e em homens }(38,1 \%) \text {; dislipidemia em homens } \\
(58,5 \%) \text { e em mulheres }(58,8 \%) \text {. }\end{array}$ \\
\hline 03 & $\begin{array}{l}\text { Média de idade de } 57,5 \text { anos; sexo masculino }(72,7 \%) \text {; cor branca } \\
(67,3 \%) \text {; ensino fundamental incompleto }(36,0 \%) \text {; renda menor que três } \\
\text { salários minimos }(58,3 \%) \text {; hipertensão arterial sistêmica }(66,7 \%) \text {; } \\
\text { dislipidemia }(40,0 \%) \text {; diabetes mellitus( } 28,7 \%) \text {; história familiar }(60,7 \%) \text {; } \\
\text { obesidade }(21,4 \%) \text {. }\end{array}$ \\
\hline 04 & 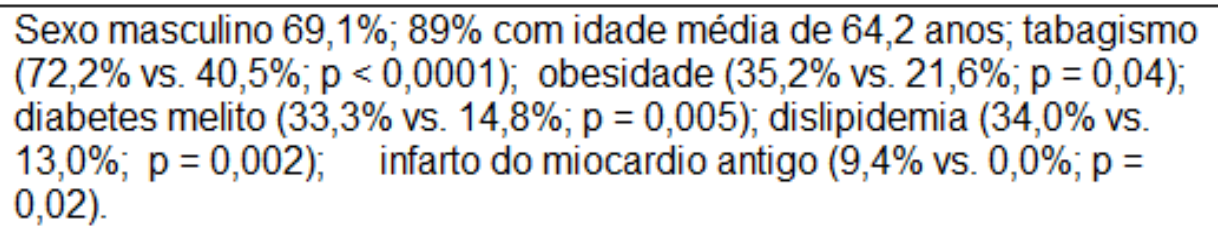 \\
\hline 05 & $\begin{array}{l}\text { Sexo masculino } 62,2 \% \text {; idade média de } 59 \text { anos; ensino fundamental } \\
\text { incompleto }(48,6 \%) \text {; renda menor que três salários mínimos }(35,1 \%) \text {; } \\
\text { hipertensão arterial }(70 \%) \text {; diabetes ( } 30 \%) \text {; dislipidemia }(58,5 \%) \text {; história } \\
\text { familiar de doença cardiaca isquêmica } 32,4 \% \text {; sedentarismo } 81 \% \text {; } \\
\text { tabagismo atual } 68 \% \text {; álcool em } 21,6 \% \text {; estresse cotidiano em } 62 \% \text {. }\end{array}$ \\
\hline 06 & $\begin{array}{l}\text { Idade entre } 36-89 \text { anos } 59 \% \text {; sexo feminino } 36,9 \% \text {; sedentarismo } \\
79,8 \% \text {; idade avançada } 79,8 \% \text {; hipertensão arterial sistêmica } 67,9 \% \text {; } \\
\text { tabagismo } 65,5 \% \text {; dislipidemia } 42,9 \% \text {; hereditariedade } 38,1 \% \text {; diabetes } \\
\text { mellitus } 33,3 \% \text {; estresse } 16,7 \text {; obesidade } 7,1 \% \text {; antecedentes de doen- } \\
\text { ça cardiaca } 34,5 \% \text {. }\end{array}$ \\
\hline 07 & $\begin{array}{l}\text { Sexo masculino } 66,7 \% ; 73,2 \% \text { na faixa } 50 \text { - } 80 \text { anos; } 71,1 \% \text { com renda } \\
\text { mensal menor que } 6 \text { salários-mínimos; } 82,2 \% \text {, analfabetos ou com } \\
\text { primeiro grau incompleto; hipertensão arterial sistêmica }(47,2 \%) ; \\
\text { diabetes mellitus( } 3,8 \%) \text {; tabagismo }(25,5 \%) \text { e etilismo }(1,9 \%) \text {. }\end{array}$ \\
\hline 08 & $\begin{array}{l}\text { Média de idade foi de } 59,9 \pm 11,55 \text { anos; sexo masculino }(81,3 \%) \text {; cor } \\
\text { branca }(83,3 \%) \text {; ensino fundamental incompleto }(72,9 \%) \text {; renda menor } \\
\text { que três salários mínimos }(58,3 \%) \text {; sedentarismo }(91,7 \%) \text {; hipertensão } \\
\text { arterial sistêmica }(63,8 \%) \text {; estresse }(50 \%) \text {; história familiar }(43,7 \%) \text {; } \\
\text { tabagismo }(41,7 \%) \text {; sobrepeso }(35,5 \%) \text {; obesidade }(33,4 \%) \text {; dislipidemia } \\
(23 \%) \text {; diabetes mellitus }(20,8 \%) \text {; consumo de álcool }(12,5 \%) \text {. }\end{array}$ \\
\hline
\end{tabular}


Figura 1 - Prevalência da citação dos fatores de risco que obtiveram uma porcentagem a partir de $30 \%$ nos estudos avaliados, Brasil, 2017.
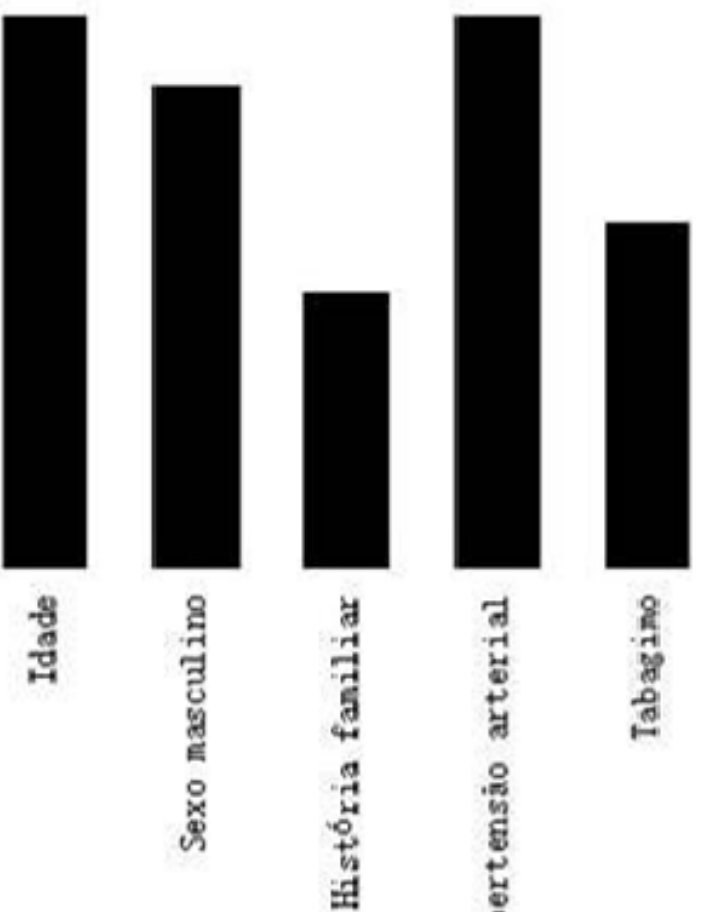

न्ل
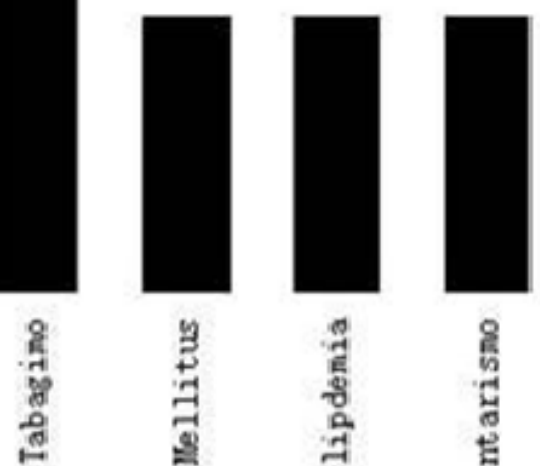

608
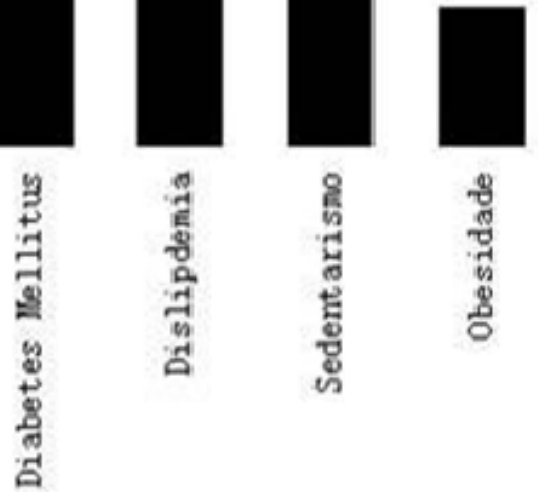

$20 x$

Ox

Porcentagen da cit æáo dos fatores de risco nos estudos avaliados:

\section{Conclusões}

O estudo mostra que a maior parte da população estudada com síndrome coronariana aguda sofre com maior influência de fatores de risco modicáveis. Os fatores de risco modificáveis mais frequentes nos estudos foram à hipertensão arterial, seguida de tabagismo.

É necessário que os profissionais da saúde avaliem os fatores de risco mais prevalentes na população a ser assistida, visando realizar uma assistência direcionada e consequentemente mais eficaz.

Pode ser considerado um fator limitante do estudo a pouca quantidade de estudos avaliados. É recomendada a realização de estudos multicêntricos em todas as regiões do país avaliando todos os fatores relacionados à SCA.

\section{Referências}

1 Schmidt MI et al. Doenças crônicas não transmissíveis no Brasil: carga e desafios atuais. THE LANCET. London, p.61-74, maio. 2011. Disponível em: < http: // download.thelancet.co $\mathrm{m} /$ flatcontentassets/pdfs/brazil/brazilpor4.p df $>$.

2 Brasil. Ministério da Saúde [Internet]. RIPSA. Indicadores e Dados Básicos - Brasil 2013. IDB 2013. [acesso em 2017 jul. 03]. Disponível em:< http://tabnet2.datasus.gov.br/cgi/idb2013/mat riz.htm>

3. Anderson $\mathrm{JL}$ et al. ACC/AHA Guidelines for the management of patients with unstable angina/non-ST segment elevation myocardial infarction: executive summary. A report of the American College of Cardiology/American Heart association Task Force on Practice Guidelines. Circulation, Kyoto, 2007; 116:148-304.

4. Giugliano RP, Braunwald E. The Year in non-ST segment elevation acute coronary syndrome. Jour. Ame. Coll. Cardio. 2009; 
54(16):1544-55.

5. Spinel LF, Püschel VAA. Perfil de estilo de vida de pessoas com doença cardiovascular. Rev.Gaú. de Enferm. 2007; 28 (4):534-41.

6. Lima FET. Araújo TL, Lopes, MVO, Silva , LF, Monteiro, ARM, Oliveira, SKP. Fatores de risco da doença coronariana em pacientes que realizaram revascularização miocárdica. Rev. Rene 2013;13(4):853-60.

7. Pereira JC, Barreto SM, Passos VMA O perfil de saúde cardiovascular dos idosos brasileiros precisa melhorar: estudo de base populacional. Arqui. Bras. de Cardio. 2008;91(1),:1-10.

8. Teston EF, Cecilio HPM, AL Santos, Arruda GO, Radovanovic CAT, Marcon, SS. Fatores associados às doenças cardiovasculares em adultos. Rev. de Med.(Ribeirão Preto) 2016; 49(2):95-102.

9. Labarthe DR, Dunbar SB. Global Cardiovascular Health Promotion and Disease Prevention: 2011 and Beyond. Circulation 2012; 125(21):2667-76.

10. Andrade PB et al. Perfil clínico e angiografico de pacientes jovens submetidos a intervenção coronaria percutanea primaria. Rev. Bras. de Cardio. Invas. 2015; 23(2):91-5.

11 Silva JLL, Souza SL. Fatores de risco para hipertensão arterial sistêmica versus estilo de vida docente. Rev. Elet. de Enferm. 2004; 6(3):330-335.

12. Gander ML, Fischer JE, Maly FE, von Känel R. Effect of the G-308A polymorphism of the tumor necrosis factor (TNF) a gene promoter site on plasmalevels of TNF $-\alpha$ and C-reactive protein in smokers: a cross-sectional study. BMC Cardiovascular Disorder. 2004; 4(17):1-7.

13. Giovino GA, Mirza SA, Samet JM, Gupta PC, Jarvis MJ, Bhala N, et al. Tobacco use in 3 billion individuals from 16 countries: an analysis of nationally representative crosssectional household surveys. Lancet. 2012; 380(9842):668-79.

14. Malta D, Oliveira TP, Santos MAS, Andrade SACA, Silva MMA. Avanços do Plano de Ações Estratégicas para o Enfrentamento das Doenças Crônicas não Transmissíveis no Brasil, 2011-2015. Epidemiol Serv Saúde 2016 Jun; 25(2): 373-90.

15. Costa MC, Fideles IC, Azevedo VBR, Meira GLS, Leite L. Fatores de risco para doenças crônicas não transmissíveis identificados em trabalhadores atendidos em um ambulatório de nutrição. Rev. Bai. Saúd. Públ. 2012;36(3):727-39.

16. Gorayeb R, Facchini, GB, Schmidt A.
Caracterização Psicossocial de Pacientes Internados em Enfermaria de Cardiologia. Rev. Bras. Cardio. 2012; 25(3): 218-25.

17. Lemosa KL et al. Prevalência de fatores de risco para síndrome coronariana aguda em pacientes atendidos em uma emergência. Rev. Gaú. de Enferm. 2010; 31(1):129-35.

18. Jesus AV, Campelo V, Silva MJS. Perfil dos pacientes admitidos com Infarto Agudo do Miocárdio em Hospital de Urgência de Teresina-PI. Rev. Interdiscip 2013;6(1):25-33.

19. Almeida $\mathrm{MC}$ et al. Comparação do Perfil Clínico-Epidemiológico entre Homens e Mulheres na Síndrome Coronariana Aguda Rev.Bras. de Cardio. 2014; 27(6):423-429.

20. Brunori EHFR et al. Associação de fatores de risco cardiovasculares com as diferentes apresentações da síndrome coronariana aguda. Rev. Lat. Amer. de Enferm. 2014; 22(4):538-46.

21 Silva PRF et al. Avaliação do retardo pré-hospitalar no cuidado ao infarto agudo do miocárdio no Centro Oeste de Minas Gerais, Brasil. Rev. de Med. de MG. 2015;25(3):353-62.

22 Santos FG et al. Avaliação da qualidade do atendimento ao paciente com síndrome coronariana aguda no serviço de emergência. Rev. Elet. de Enferm. 2015; 17(4):19.

23. Araújo IFM et al. Perfil da população acometida por infarto agudo do miocárdio. Rev. Enferm. UFPE on line 2016;10(7):2302-09.

24. Mertins SM et al. Prevalência de fatores de risco em pacientes com infarto agudo do miocárdio. Avan. em Enferm. 2016;34(1):30-8.

\section{Endereço para Correspondência}

Faculdade Estácio de Sergipe

Rua Teixeira de Freitas, 10 - Salgado Filho, Aracaju - SE

CEP.: 49020-490

e-mail: miriageisaenf@gmail.com

Recebido em 21/08/2017

Aprovado em 05/02/2018

Publicado em 11/05/2018 